\title{
Ultra-high C-reactive protein levels in hospitalized inflammatory bowel disease (IBD) patients predicts serious complications
}

\author{
Kofi Clarke • Jana G. Hashash • Jason M. Swoger • \\ Miguel Regueiro
}

\section{Dear Editor:}

C-reactive protein (CRP), erythrocyte sedimentation rate, fecal lactoferrin, and fecal calprotectin have been evaluated as markers of disease activity and predictors of disease course in inflammatory bowel disease (IBD). Studies on the predictive value of CRP elevations in IBD patients are difficult to interpret or generalize because of different laboratory cutoff values.

Fourteen hospitalized IBD patients with ultra-high Creactive protein (UH-CRP), defined as CRP more than ten times the upper limit of normal (ULN), were identified and followed for 6 months. Eight patients had ulcerative colitis, ages ranged between 20 and 69 years, with a mean of $37.36 \pm 17.248$ (SD) years. CRP levels ranged from 14.3 to 41 times ULN, with a mean elevation of 25.66 times the $\mathrm{ULN} \pm 8.67$ (SD).

Ten out of 14 patients $(71.43 \%)$ had IBD-related complications, with $50 \%$ requiring surgery either during admission or within 6 months. Three out of five patients who were admitted with typical symptoms of abdominal pain and bloody diarrhea had severe complications, two had a perforated viscus and one had multiple intraabdominal abscesses. One in five had a bowel obstruction, and two $(14.3 \%)$ had a newly diagnosed fistula. Finally, three patients $(21.4 \%)$ had an acute severe infection; two with clostridium difficile associated colitis, and the third with enterococcus bacteremia. Compared to previously reported data, there was a non-statistically significant trend towards longer hospital stay in patients with UH-CRP elevations.

A UH-CRP level in hospitalized IBD patients is associated with significant complications and should not be solely attributed to disease flare. Describing CRP elevations as multiples of the upper limit of normal from various laboratories provides an alternative way of comparing results, and may help predict serious complications. Additional prospective studies are needed.

K. Clarke $(\bowtie) \cdot J$. G. Hashash · J. M. Swoger · M. Regueiro West Penn Allegheny Health System and University of Pittsburgh Medical Center, Pittsburgh, PA, USA

e-mail: kclarke@wpahs.org 